\title{
Optimization of Energy Consumption in Cognitive Radio Networks based on Renewable Energies.
}

\author{
Víctor Gomez ${ }^{1}$, Camila Salgado ${ }^{2}$ and Cesar Hernandez ${ }^{3}$ \\ ${ }^{1}$ Esp. Electrical Engineer, Schneider Electric, Colombia. \\ ${ }^{2}$ MSc. Researcher, Universidad Distrital Francisco José de Caldas, Colombia, \\ ${ }^{3}$ PhD Titular professor, Universidad Distrital Francisco José de Caldas, Colombia.
}

\begin{abstract}
Energy consumption is one of the most important aspects in wireless communications where networks and nodes exchange transmission and reception parameters, that ultimately results in changes in consumption parameters. This is specifically related to the offer and demand profiles seen in the electric supply chain. Said changes are proportional to the number of channels and users that interact in the radio-electric spectrum. Therefore, the present article examines energy-efficient cognitive radio techniques and the optimization of wireless networks fed by non-conventional energy sources. Seeing green energies as an important resource in the future, the performance of the network strongly depends on the spectrum dynamics and the energy available. In contrast with traditional energy sources, the rate of arrival of green energies is dependent on the recollection systems and is marked by randomness and intermittence. To optimize and adapt the use of energy according to the availability of the opportunistic spectrum, the main challenges faced in the design of cognitive radio networks that operate under different storage sources whose applicability is analog to the distributed energy resources.
\end{abstract}

Keywords: Cognitive Radio; Efficient Spectrum, Energy, Telecommunications.

\section{INTRODUCTION}

The accelerated digital interconnection pacing everyday life has brought forth an exponential increase in the construction, development and adaptation of the communications infrastructure, so the current consumption rate has been augmented leading to fast-paced and ever-growing energy consumption [1][2]. Hence, different research projects have aimed their efforts at verifying all the aspects going from the optimization of the radio spectrum up tothe adoption of energyrelated strategies that seek to cut CAPEX and OPEX expenses as well as improve the sustainability of information and communications technologies (ICTs) making use of selfsustainable systems [3][4].

The most eco-friendly wireless access networks can capitalize on the wide paradigm of Cognitive Radio (CR). Haykin [5] defines CR as: "a cognitive radio transmitter will learn from its environment and adapt its internal states to the statistical variations of the radio frequency stimuli, by adjusting its transmission parameters (for instance, frequency band, modulation mode and transmission power) in real time and online". The cognitive capacity to detect the available spectrum and the reconfigurability to dynamically access the spectrum over which the experiments are carried out with less fading and interference events, enable the smart CR system to improve the spectrum agility and energy efficiency [6][5].

Among the many areas of wireless systems that can be improved with $\mathrm{CR}$, the existing literature focuses on improving the efficiency of the spectrum instead of energy usage and focus even less on unreliable dynamic energy [7]. Hence, Zhao and Sadler [8] presented a general description of the Dynamic Spectrum Access (DSA) that differentiates the wireless CR system from inherited wireless systems, that are licensed to operate within a given frequency band. The research draws the first indications that quality in data services is intimately tied to the quality and continuity of the electric service for rural and urban areas [9].

The previous conjuring presents the paradigm of reflecting on current CR networks fed by a reliable energy source that can be either rechargeable or non-rechargeable, so the continuous advances in green energy have prompted us to focus on energy efficiency and studying the green powered cognitive radio network [10][11]. In agreeance with this statement, the concept of recollecting energy to capture and store green energies to generate electricity or other form of energy, which is renewable and more eco-friendly than the one derived from fossil fuels [12][13][14]. If the green energy is wide and stable in the sense of availability, cognitive radio can be powered to harness the underused spectrum through the free energy without the need of an external energy supplement from the power grid or selfsupply batteries of the system (See Figure 1).

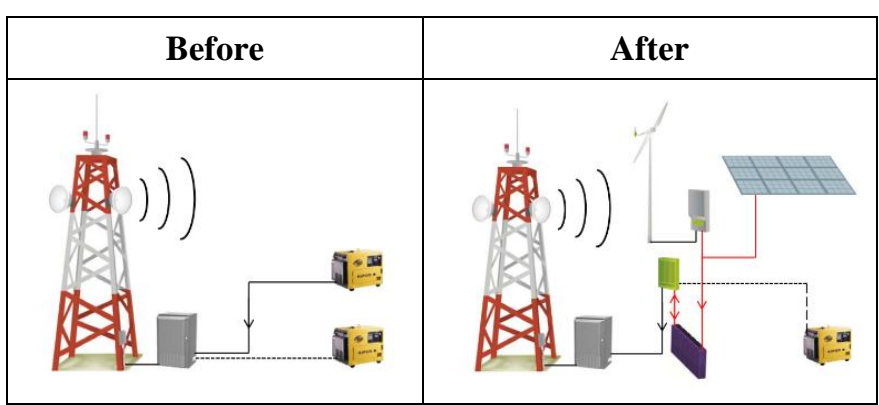

Fig. 1. Base unit powered by a (a) Diesel generator, and (b) wind generator and photovoltaic solar panel. 
However, it is not trivial to design and optimize CR networks enabled for green energies due to the opportunistic use of the inactive sections of the spectrum and free energies. This review summarizes the avant-garde research in energy-efficient cognitive radio systems based on three aspects:

- Achieving a power-aware functionality within systems for CR

- Designing energy-efficient wireless access systems through cognitive radioelectric

- Optimizing green networks in $\mathrm{CR}$

Although green energy wireless networks are not currently implemented on a large scale due to the higher cost per Watt (W) in comparison to the network energy, feeding wireless networks with green energy is imminent and is becoming an eco-friendly sustainable solution. The purpose of this article is to provide some ideas for future research in cognitive radio based on dynamic spectrum access that are capable of releasing wireless access networks from spectrum-related constraints and specially from energy-related restrictions.

The present document synthesizes the most important details in research developed in [15], organized as follows: after returning to the main concepts of cognitive radio and dynamic spectrum access, a summary is offered of the detection of energy efficiency, management and transfer of the spectrum, and the spectrum trade methods used in cognitive radio. This is followed by 2) energy supply models available in communication networks and in 3) the optimization of green energy usage in Cognitive Radio Networks - CRN along with open research subjects.

\section{GENERAL FRAMEWORK OF CR}

The concept of CR was developed by Joseph Mitola III in 1999 as the "point in which the wireless Personal Digital Assistant (PDA) and related networks are in computational terms, smart enough compared to radio resources and the corresponding communications between computers, to be able to detect the incoming communication needs of the user as a function of the usage context and provide the most adequate wireless resources in that same instant2"[16].

Based on the first definition, the cusp of what is known as optimization began, seen as giving a more efficient use to the radio spectrum, through a dynamic allocation that is adjusted in real time to changes in the communications network. Hence, considering the classification of Primary Users (PU) and Secondary Users (SU), the PU are those who have a priority in the use of channels as telecommunication routes while the SU are those who opportunistically use the frequency band. In other words, the term opportunistic refers to the capacity to employ the communication channel when the PU requires it for its use. This use is also supported legally by services providers.

\section{ENERGY EFFICIENCY IN CR}

Traditional spectrum licences use the command and control model, in which explicit rules are established for spectrum usage [17]. The underuse of the spectrum with a static license represents significant challenges for wireless applications and new devices. Then, in order to allow a more flexible access to the spectrum, dynamic access techniques have been proposed with the purpose of solving inefficiency matters and allowing unlicensed users to access the spectrum under certain constraints.

In a dynamic access network, three models are commonly used based on [8][17]). Cognitive radio technology has become a key enabler for dynamic access to the spectrum. In a DSA network based on CR, the primary system possesses the rights on the spectrum while unlicensed users can dynamically share the licensed spectrum in an opportunistic manner. This capacity is provided by the following cognitive functionalities [18].

\section{III.I Spectrum detection and analysis}

To validate the algorithm, the transactional database (TD) is obtained from the private key (PK). The TD contains specific data such as origin and destination IPs while a software called ALICE obtains the data from equations (1) and (2).

\section{III.II Spectrum detection and analysis}

Spectrum detection is defined as the task of finding spectral opportunities, i.e. holes in the spectrum around the vicinity of the overlapped CR receiver [19]. Once the secondary unlicensed transmitter uses the spectrum hole, this secondary receiver will not affect any primary receiver and no primary transmitter will interfere with the expected secondary receiver [5]. The spectrum analysis uses the information obtained from the detection process to program and make a decision to access the spectrum through SU.

\section{III.III Spectrum management and transfer}

This function allows SU to choose the best available channel and release the channel when the PU wishes to seize it again [20][21], and it also takes on the challenges imposed by the fluctuating nature of the spectrum such as the different requirements of Quality of Service (QoS) from various applications [22].

\section{III.VI Allocation and shared use of the spectrum}

It coordinates the access to the channels available with other users (PU, other SU or both) so the level of induced interference for the use of the secondary spectrum must be maintained below a certain threshold and collisions and interferences are tackled for multiple SU.

Although CR allows to share the spectrum with smart strategies and an easy access [23], an important limitation of a practical cognitive radio network is the increase in energy consumption introduced by the cognitive capacity and re-configuration. The 
cognitive radio system aware of the energy demand has been researched from three general perspectives:

- The minimization of energy lowers energy consumption for the given requirements:

- The maximization of performance increases performance for a limited energy budget;

- The maximization of utility takes into account the cost of energy consumption during the detection and/or transmission with utility functions with given rates between the performance reward and the energy costs.

Regardless of the spectrum exchange model, the spectrum allocation mechanisms have a significant impact in energy consumption and the performance of each individual SU and the entire secondary network. As a result, energy-efficient designs that consider the diversity of SU energy assumptions, channel conditions and QoS requirements, are intertwined with spectrum allocation and distribution algorithms.

\section{III.V Design Problem of the Detection Architecture:}

As shown in Table 1 multiple SU detect and share information such as the energy consumed by the Sensorial Power (PS) detection and the notification of the consumed power (PR) can be reduced when each cognitive radio randomly turns off the detection device. This energy saving approach is called sleep or turn on/off detection. The disconnection probability is called the sleep rate [24].

The second approach to reduce PR is the censure, where the detection result is only sent if it is considered to be informative. The censure rate refers to the probability that the detection results are within the censure region, defined as a signal interval for the energy collected locally [30]. Another method to reduce PR is grouping: instead of sending local detection results directly to the fusion center (FC), they are sent to the Allocated Grouping headers $(\mathrm{CH})$ that make decisions of local grouping and send them to the FC. Hence, the network energy consumption is reduced due to the reduction in distance. The optimization algorithms of centralized detection performance and general expense minimization can be found in [31][32], where three approaches are implemented in a complementary manner. In addition to the centralized programming of spatial diversity, each SU can decide by himself whether to sleep or share the results. However, the transmission nature of the wireless environment gives the opportunity to the SU to become a pilot for free, that listens to the detection result and does not contribute to the detection process. Different games have been modeled [33][34] to study the incentive strategy previously mentioned of the cooperative spectrum detection where each selfish SU intends to maximize their own utility.
Table 1. Spectrum categories in terms of energy consumption

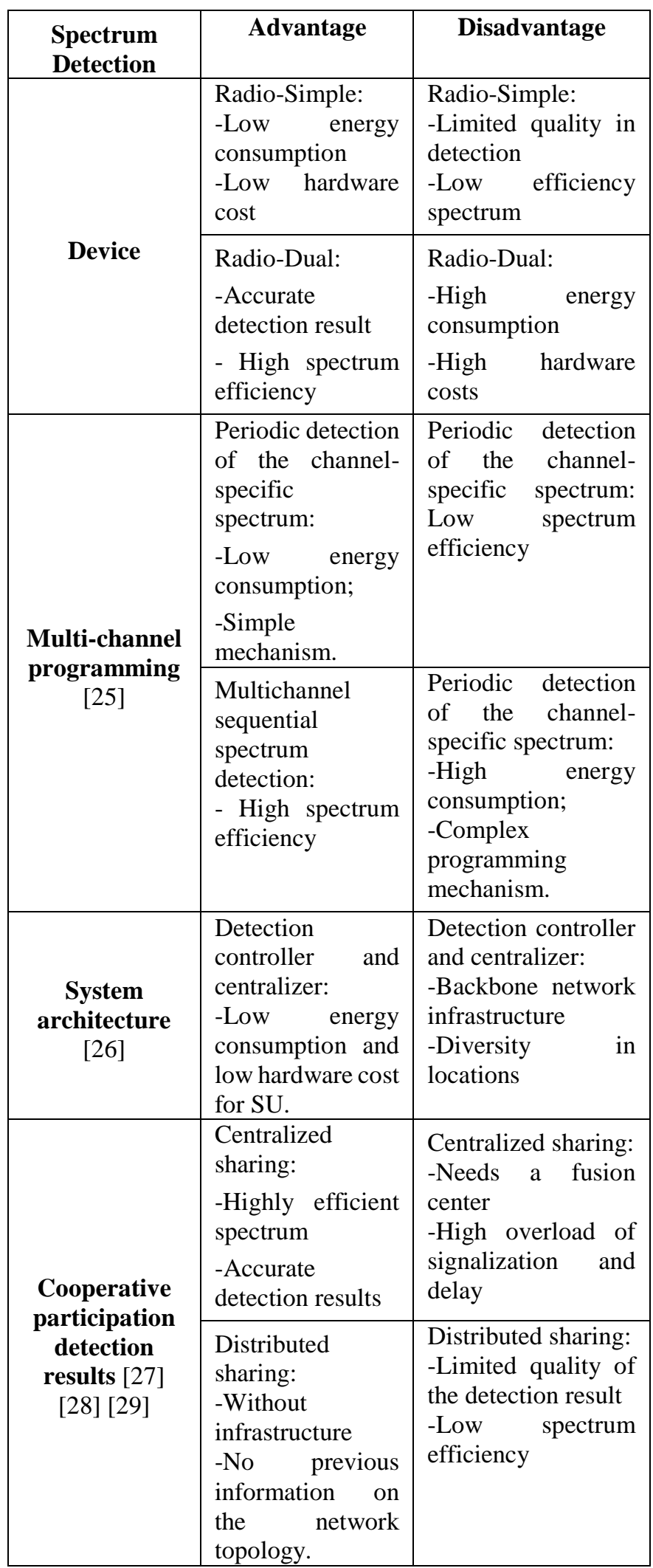




\section{GREEN POWERED COGNITIVE RADIO NETWORKS}

In this section, the adoption of $\mathrm{CR}$ in wireless networks is discussed to improve the energy efficiency. Given the complexity of the subject and the diversity of the existing technical approaches [35], the main applications of communications in cooperative and cognitive radio are analyzed and in heterogeneous cognitive radio for long-term evolution emerging networks (LTE-Advanced).

Therefore, although the spectrum efficiency based on cognitive radio has been discussed, the integration of renewable resources in terms of energy supply substantially improves the continuity and reliability of communication systems. Thus, as smart grids are developed, non-conventional sources also move forward to face the energy demands. For instance, solar and wind energies can significantly reduce the carbon footprint and in parallel contribute to the harnessing of the radio electric spectrum. Furthermore, the penetration of distributed energy resources (DER) is having an exponential increase worldwide. In a nearby future, consumers can contribute with clean energy to the network [36][37].

Distributed power generators, starting from users known as prosumers (consumer of a product of service that also produces it), are at the forefront of the development to harness power micro-networks. Hence, telecommunication manufacturers such as Nokia, Siemens, Huawei and Ericsson have designed and built stations outside of the network that are powered by green energies to reduce the OPEX of mobile networks in rural areas [38]. The general energy consumption of the wireless network depends on the power generated by the environment, the storage capacity of the battery, the traffic demands and the conditions of the wireless channel [39]. which are all dynamic processes as shown in Figure 2.
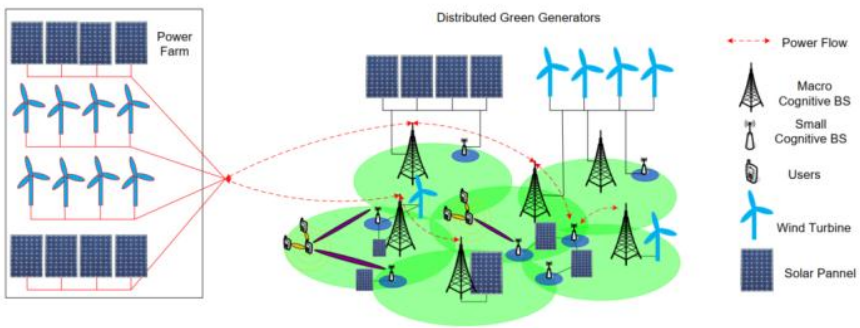

Fig. 2. Green supply chain for cognitive radio networks [15]

In addition to the use of independent power generators, the optimization of cognitive radio networks enabled for green energies to share and commercialize energy with other DER generators supposes an articulation of telecommunications and energy resources seeking to guarantee a sustainable network of communications in urban and rural areas. Figure 2 illustrates the supply models based on clean energies.

In comparison to the cellphone networks powered by green distributed generators or green energy farms, the operation of cognitive radio networks powered by green energies is more complex due to the following reasons:

- Different system architectures require a different energy distribution within the network. For instance, the centralized controller requires more energy in the spectrum detection phase, while the SU demand more energy in the data transmission phase;

- The cognitive functionality depends on energy availability, while the spectrum availability affects energy consumption [40]. To untangle the intertwined energy and the spectrum dynamics within the complex network architectures, it is first discussed how to optimize the use of green energies within the cellphone network that only factors in the energy dynamics. This grants some ideas for data transmission in green cognitive radio. Afterwards, with the introduction of the spectrum dynamics, the use of energy is studied in green cognitive radio networks.

\section{IV.I Wireless network implemented with Distributed Power Generators.}

Taking advantage of the characteristics of wireless communications can facilitate the exchange of energy with neighboring networks through traffic download. Different schemes of user association to the Base Station (BS) or cellsized adaptation schemes can be sued in this method implicitly. Hence, Han and Ansari [41] [42] considered the centralized user association scheme by taking into account green energy storage and the traffic queue of multiple base stations. This leads to an energy-conscious scheme with a latency that minimizes the pondered sum of traffic from $\mathrm{BS}$ in a heterogeneous mobile network. Meanwhile, the user can be associated to multiple base stations as seen in the Coordination of Multi-Point (CoMP) [43], transmission so that the channel conditions, traffic load and amount of residual green energy can be used conjointly for data transmission. Similarly, the cellsized adaptation technique allows BS to decide to expand and cover more active users or reduce their size until the enter a sleep state.

With the network infrastructure, the power generated by distributed energy resources can be shared directly. For instance, when traffic users are far from the base stations with wide energy storage, the traffic download is not affordable nor even feasible due to the fading of the wireless channel. In this case, it is more rational to transmit energy directly through the supply chain. Furthermore, the energy trade can be implemented in the node with traffic to serve and the node with wide energy storage can be selfish with the user. The commercial process can also take the shape of traffic download, power transmission or both [37].

\section{IV.II Wireless network powered by green energy farms}

Although energy can be shared or exchanged as a fundamental resource that determines the performance of the wireless system, there is a side effect for energy and interference. Less power means less interference for neighbors. As a consequence, the power from neighbors will be less consumed. Based on this scenario, Kwak et al. [44] implemented the centralized energy distribution system through interference management. 
For a given energy budget destined to buy energy from the provider, or for a given capacity of energy farm, the allocation of energy to multiple BS and time slots determines the longterm utility within the network. Utility based on the signal-tointerference-plus-noise ratio (SINR) is optimized through user programming and power control of the BS for different restraints of shared power usage in space and time.

When the smart network that uses green power, plants is the energy provider for the wireless cellphone network, the energy price has a great impact in the energy efficiency of the cellphone network. Due to the uncontrollable nature of the energy source, the generated green energy can become a surplus and damage the energy providers given that the storage costs will affect the income [45]. As a result, balancing out the market with a pricing strategy is extremely important when supply systems are used with clean energies.

Research carried out by Bu et al. [46] proposed a system with multiple electricity retailers and multiple BS groups. The BS within a group can be left in stand-by or remain active and participate in the CoMP transmission with other active members of the group. Keeping in mind the level of contaminants and the price offered for each retailer, the independent groups can decide from which retailer to acquire electricity and the forecast of power demand. Based on the previous statement, the system has been modeled as a Stackelberg game with two levels, where 1) the Stackelberg leaders, the power retailers try to maximize income; 2) the Stackelberg follower in the cellphone network tries to maximize the utility function in terms of the blockage probability, the energy cost and the levels of contaminants (see Figure 3).

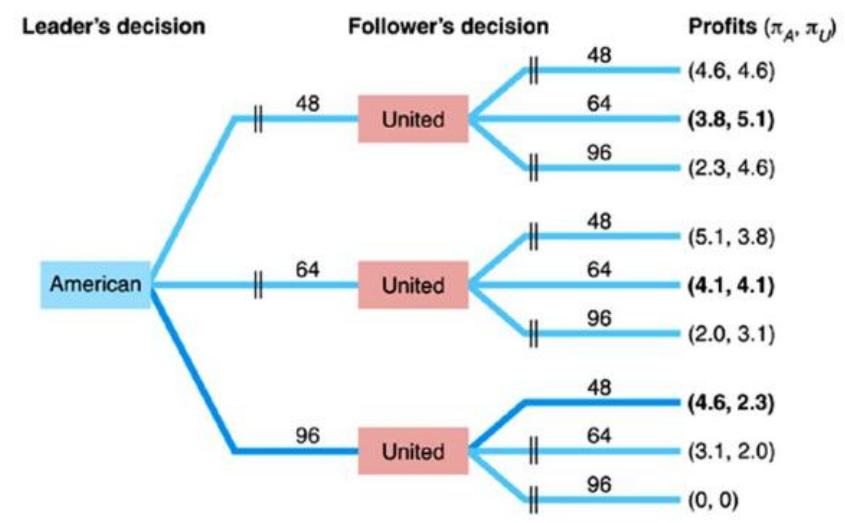

Fig. 3. Stackelberg game theory.

\section{IV,III Cognitive radio network powered by green energy}

The wireless network enabled for green energy can be supported by non-conventional power sources or distributed power generators. The Table 2 describes the pros and cons of these types of supports. In particular, green energy is more expensive to build while the energy provided by distributed generators is less stable [47].
Table 2. Green energy strategies for wireless communication networks. [15]

\begin{tabular}{|l|l|l|}
\hline $\begin{array}{c}\text { Energy of the } \\
\text { generator }\end{array}$ & \multicolumn{1}{|c|}{$\begin{array}{c}\text { Green energy } \\
\text { farm }\end{array}$} & \multicolumn{1}{|c|}{$\begin{array}{c}\text { Distributed } \\
\text { generators }\end{array}$} \\
\hline Scala & $\begin{array}{l}\text { Large and remote } \\
\text { energy provider }\end{array}$ & $\begin{array}{l}\text { Local and small } \\
\text { energy provider }\end{array}$ \\
\hline $\begin{array}{l}\text { Energy storage } \\
\text { unit }\end{array}$ & High capacity & Low capacity \\
\hline Available energy & More stable & Less stable \\
\hline $\begin{array}{l}\text { Energy transfer } \\
\text { method }\end{array}$ & Network structure & $\begin{array}{l}\text { Directly connected to } \\
\text { a BS }\end{array}$ \\
\hline $\begin{array}{l}\text { Energy shared } \\
\text { with the network }\end{array}$ & $\begin{array}{l}\text { Network structure or } \\
\text { traffic download }\end{array}$ & $\begin{array}{l}\text { Network structure or } \\
\text { traffic download }\end{array}$ \\
\hline
\end{tabular}

Whether it is powered by a green energy farm or distributed generators, the cognitive radio network poses more challenges for green energy usage since the nodes with an available spectrum, the nodes with enough energy and those with useful traffic data may all be different. The emerging methods that can balance the spectrum, the power and the traffic within the secondary network are desirable and briefly analyzed.

- Sharing and/or exchanging detection results is a power balance method. For instance, traffic reaches a SU transmitter that is supposed to detect the spectrum and carry out the data transmission to the chosen SU receiver. A third $\mathrm{CR}$ node close to the $\mathrm{SU}$ receiver can continue with the detection and offer the detection results as long as the SU transmitter is scarce on energy. A sufficient amount of energy allows the CR node to even download the data traffic from the SU transmitter. Thus, the transmission power is saved due to a shorter distance between the third CR node and the SU receiver. On another note, if all the cognitive radio devices act as a backup storage energy unit, the time-space energy exchange is carried out within the networks.

- The mobile network based on wireless power transfer is another approach that can balance out the power within the cognitive radio network. The mobile vehicles / robots that carry high volume batteries, can serve as back storage of mobile energy and periodically deliver energy to cognitive devices with insufficient power supply. [48]

- The power balance can depend on the network architecture to transmit energy, especially when the energy source and the cognitive devices are very distant from each other. Given that the spectrum opportunity is a local concept, downloading the detection tasks or exchanging the detection results is not reliable. Furthermore, moving vehicles through long distances will lead to high energy consumption. 
International Journal of Engineering Research and Technology. ISSN 0974-3154, Volume 13, Number 8 (2020), pp. $1999-2006$

(C) International Research Publication House. https://dx.doi.org/10.37624/IJERT/13.8.2020.1999-2006

\section{RECOMMENDATIONS}

The increase in the number of devices connected to the communication networks, along with the exponential demand in the data usage of PU and SU has brought forth a vertiginous interest in the study of new techniques to render the usage of the radio spectrum more efficient. Nonetheless, it is important that said research projects are linked to the efficient use of power with supply-oriented methodologies.

The main recommendation would consist on using optimization methods to articulate an efficient use of the spectrum supported on the energy supply based on centralized or distributed smart grids.

\section{CONCLUSIONS}

The energy consumption is one of the most important aspects in mobile and wireless communications. The present review evidenced that there are research ventures that have proven the significant advantages of powering said communication architectures through renewable energy sources. This represents savings in terms of operational costs and an increase in efficiency compared to the dynamic changes present among primary and secondary users, when interacting within the cognitive radio network.

The implementation of Green Energy farms in a centralized or distributed manner requires drastic investment at the beginning of projects, although they could have medium and long-term benefits in returning the investment. Additionally, there is an invaluable advantage in improving the customer experience in terms of QoS satisfaction. A clean energy system backed by different supply sources offers higher levels of reliability in the power demand from base stations in rural and urban perimeters.

\section{ACKNOWLEDGMENTS}

The authors wish to thank the Center for Research and Scientific Development of the Universidad Distrital Francisco Jose de Caldas for the support and financing of this research.

\section{REFERENCES}

[1] T. Han and N. Ansari, "On greening cellular networks via multicell cooperation," Wirel. Commun. IEEE, vol. 20, pp. 82-89, 2013.

[2] A. De Domenico, E. C. Strinati, and A. Capone, "Enabling green cellular networks: A survey and outlook," Comput. Commun., vol. 37, pp. 5-24, 2014.

[3] B. Wang and K. J. R. Liu, "Advances in cognitive radio networks: A survey,” IEEE J. Sel. Top. Signal Process., vol. 5, no. 1, pp. 5-23, 2010.

[4] Y. Wu and D. H. K. Tsang, "Energy-efficient spectrum sensing and transmission for cognitive radio system," IEEE Commun. Lett., vol. 15, no. 5, pp. 545-547, 2011.

[5] S. Haykin, "Cognitive radio: brain-empowered wireless communications," Sel. Areas Commun. IEEE J., vol. 23, no. 2, pp. 201-220, 2005.

[6] J. Mitola, "Cognitive radio---an integrated agent architecture for software defined radio," 2000.

[7] L. Chhaya, P. Sharma, A. Kumar, and G. Bhagwatikar, "Integration of cognitive radio with heterogeneous smart grid communication architecture," in Intelligent Communication, Control and Devices, Springer, 2018, pp. 981-989.

[8] Q. Zhao and A. Swami, "A survey of dynamic spectrum access: signal processing and networking perspectives," DTIC Document, 2007.

[9] M. Ozger, O. Cetinkaya, and O. B. Akan, "Energy harvesting cognitive radio networking for IoT-enabled smart grid," Mob. Networks Appl., vol. 23, no. 4, pp. 956-966, 2018.

[10] T. Yücek and H. Arslan, "A survey of spectrum sensing algorithms for cognitive radio applications," Commun. Surv. Tutorials, IEEE, vol. 11, no. 1, pp. 116-130, 2009.

[11] H. Sun, A. Nallanathan, C.-X. Wang, and Y. Chen, "Wideband spectrum sensing for cognitive radio networks: a survey," IEEE Wirel. Commun., vol. 20, no. 2, pp. 74-81, 2013.

[12] T. Le, K. Mayaram, and T. Fiez, "Efficient far-field radio frequency energy harvesting for passively powered sensor networks," IEEE J. Solid-State Circuits, vol. 43, no. 5, pp. 1287-1302, 2008.

[13] S. Alam, M. F. Sohail, S. A. Ghauri, I. M. Qureshi, and N. Aqdas, "Cognitive radio based smart grid communication network," Renew. Sustain. Energy Rev., vol. 72, pp. 535-548, 2017.

[14] H. Jabbar, Y. S. Song, and T. T. Jeong, "RF energy harvesting system and circuits for charging of mobile devices," IEEE Trans. Consum. Electron., vol. 56, no. 1, pp. 247-253, 2010.

[15] X. Huang, T. Han, and N. Ansari, "On green-energypowered cognitive radio networks," IEEE Commun. Surv Tutorials, vol. 17, no. 2, pp. 827-842, 2015.

[16] C. Augusto Hernández Suárez, I. Patricia, P. Parra, D. Armando, and G. Ramírez, Modelo adaptativo multivariable de handoff espectral para incrementar el desempeño en redes móviles de radio cognitiva. Bogotá, Colombia, 2017.

[17] M. M. Buddhikot, "Understanding dynamic spectrum access: Models, taxonomy and challenges," in 2007 2nd IEEE International Symposium on New Frontiers in Dynamic Spectrum Access Networks, 2007, pp. 649-663.

[18] B. Wang and K. J. Liu, "Advances in cognitive radio networks: A survey," Sel. Top. Signal Process. IEEE J., vol. 5, no. 1, pp. 5-23, 2011.

[19] S. Haykin, D. J. Thomson, and J. H. Reed, "Spectrum sensing for cognitive radio," Proc. IEEE, vol. 97, no. 5, pp. 849-877, 2009. 
[20] A. S. Cacciapuoti, I. F. Akyildiz, and L. Paura, "Primaryuser mobility impact on spectrum sensing in cognitive radio networks," in IEEE International Symposium on Personal, Indoor and Mobile Radio Communications, PIMRC, 2011, pp. 451-456.

[21] R. C. Qiu et al., "Cognitive radio network for the smart grid: Experimental system architecture, control algorithms, security, and microgrid testbed," IEEE Trans. Smart Grid, vol. 2, no. 4, pp. 724-740, 2011.

[22] I. F. Akyildiz, W.-Y. Lee, M. C. Vuran, and S. Mohanty, "A survey on spectrum management in cognitive radio networks," Commun. Mag. IEEE, vol. 46, no. 4, pp. 4048, 2008.

[23] G. Gür and F. Alagoüz, "Green wireless communications via cognitive dimension: An overview," IEEE Network, vol. 25, no. 2. pp. 50-56, Mar-2011.

[24] R. Deng, S. He, J. Chen, J. Jia, W. Zhuang, and Y. Sun, "Energy-efficient spectrum sensing by optimal periodic scheduling in cognitive radio networks," IET Commun., vol. 6, no. 6, pp. 676-684, 2012.

[25] H. ElSawy and E. Hossain, "Two-tier HetNets with cognitive femtocells: Downlink performance modeling and analysis in a multichannel environment," IEEE Trans. Mob. Comput., vol. 13, no. 3, pp. 649-663, 2013.

[26] K. Illanko, M. Naeem, A. Anpalagan, and D. Androutsos, "Low complexity energy efficient power allocation for green cognitive radio with rate constraints," in 2012 IEEE Global Communications Conference (GLOBECOM), 2012, pp. 3377-3382.

[27] P. Li, S. Guo, Z. Cheng, and A. V Vasilakos, "Joint relay assignment and channel allocation for energy-efficient cooperative communications," in 2013 IEEE Wireless Communications and Networking Conference (WCNC), 2013, pp. 626-630.

[28] C. Luo, G. Min, F. R. Yu, M. Chen, L. T. Yang, and V. C. M. Leung, "Energy-efficient distributed relay and power control in cognitive radio cooperative communications," IEEE J. Sel. Areas Commun., vol. 31, no. 11, pp. 2442-2452, 2013.

[29] B. Cao, Q. Zhang, and J. W. Mark, "Optimal communication strategies in cooperative cognitive radio networking," in Cooperative Cognitive Radio Networking, Springer, 2016, pp. 73-93.

[30] E. Axell, G. Leus, and E. G. Larsson, "Overview of spectrum sensing for cognitive radio," in 2010 2nd International Workshop on Cognitive Information Processing, 2010, pp. 322-327.

[31] L. Wang, J. Wang, G. Ding, F. Song, and Q. Wu, "A survey of cluster-based cooperative spectrum sensing in cognitive radio networks," in Proceedings of 2011 Cross Strait Quad-Regional Radio Science and Wireless Technology Conference, 2011, vol. 1, pp. 247-251.

[32] J. Wei and X. Zhang, "Energy-efficient distributed spectrum sensing for wireless cognitive radio networks," in 2010 INFOCOM IEEE Conference on Computer Communications Workshops, 2010, pp. 1-6.

[33] B. Wang, K. J. Liu, and T. C. Clancy, "Evolutionary cooperative spectrum sensing game: how to collaborate?," Commun. IEEE Trans., vol. 58, no. 3, pp. 890-900, 2010.

[34] J. Lai, E. Dutkiewicz, R. P. Liu, and R. Vesilo, "Comparison of cooperative spectrum sensing strategies in distributed cognitive radio networks," in 2012 IEEE Global Communications Conference (GLOBECOM), 2012, pp. 1513-1518.

[35] J. Wang, M. Ghosh, and K. Challapali, "Emerging cognitive radio applications: A survey," IEEE Commun. Mag., vol. 49, no. 3, pp. 74-81, 2011.

[36] C.-H. Lo and N. Ansari, "The progressive smart grid system from both power and communications aspects," IEEE Commun. Surv. Tutorials, vol. 14, no. 3, pp. 799$821,2011$.

[37] W. El-Khattam and M. M. A. Salama, "Distributed generation technologies, definitions and benefits," Electr. power Syst. Res., vol. 71, no. 2, pp. 119-128, 2004.

[38] T. Han and N. Ansari, "Powering mobile networks with green energy," IEEE Wirel. Commun., vol. 21, no. 1, pp. 90-96, 2014.

[39] A. He et al., "Minimizing energy consumption using cognitive radio," in 2008 IEEE International Performance, Computing and Communications Conference, 2008, pp. 372-377.

[40] W. Chung, S. Park, S. Lim, and D. Hong, "Spectrum sensing optimization for energy-harvesting cognitive radio systems," IEEE Trans. Wirel. Commun., vol. 13, no. 5, pp. 2601-2613, 2014

[41] Tao Han and N. Ansari, "Green-energy Aware and Latency Aware user associations in heterogeneous cellular networks," 2014, pp. 4946-4951.

[42] T. Han and N. Ansari, "Network utility aware traffic loading balancing in backhaul-constrained cache-enabled small cell networks with hybrid power supplies," arXiv Prepr. arXiv1409.8267, 2014.

[43] X. Huang and N. Ansari, "Joint spectrum and power allocation for multi-node cooperative wireless systems," IEEE Trans. Mob. Comput., vol. 14, no. 10, pp. 2034 2044, 2015.

[44] J. Kwak, K. Son, Y. Yi, and S. Chong, "Greening effect of spatio-temporal power sharing policies in cellular networks with energy constraints," IEEE Trans. Wirel. Commun., vol. 11, no. 12, pp. 4405-4415, 2012.

[45] F. Genoese, M. Genoese, and M. Wietschel, "Occurrence of negative prices on the German spot market for electricity and their influence on balancing power markets," in 2010 7th International Conference on the European Energy Market, 2010, pp. 1-6.

[46] S. Bu, F. R. Yu, Y. Cai, and P. X. Liu, "Dynamic operation of $\mathrm{BSs}$ in green wireless cellular networks 
International Journal of Engineering Research and Technology. ISSN 0974-3154, Volume 13, Number 8 (2020), pp. 1999-2006

(C) International Research Publication House. https://dx.doi.org/10.37624/IJERT/13.8.2020.1999-2006

powered by the smart grid," in 2012 IEEE Global Communications Conference (GLOBECOM), 2012, pp. 5201-5205.

[47] J. A. Carr, J. C. Balda, and H. A. Mantooth, "A survey of systems to integrate distributed energy resources and energy storage on the utility grid," in 2008 IEEE Energy 2030 Conference, 2008, pp. 1-7.

[48] S. Zhang, J. Wu, and S. Lu, "Collaborative mobile charging for sensor networks," in MASS 2012 - 9th IEEE International Conference on Mobile Ad-Hoc and Sensor Systems, 2012, pp. 84-92. 